\title{
Rescue of retinal degeneration by intravitreally injected adult bone marrow-derived lineage-negative hematopoietic stem cells
}

\author{
Atsushi Otani, ${ }^{1}$ Michael lan Dorrell, ${ }^{1}$ Karen Kinder, ${ }^{1}$ Stacey K. Moreno, ${ }^{1}$ Steven Nusinowitz, ${ }^{2}$ \\ Eyal Banin, ${ }^{1}$ John Heckenlively, ${ }^{2}$ and Martin Friedlander ${ }^{1}$
}

1Department of Cell Biology, The Scripps Research Institute, La Jolla, California, USA. 'Department of Ophthalmology, Jules Stein Eye Institute, UCLA School of Medicine, Los Angeles, California, USA.

\begin{abstract}
Inherited retinal degenerations afflict 1 in 3,500 individuals and are a heterogeneous group of diseases that result in profound vision loss, usually the result of retinal neuronal apoptosis. Atrophic changes in the retinal vasculature are also observed in many of these degenerations. While it is thought that this atrophy is secondary to diminished metabolic demand in the face of retinal degeneration, the precise relationship between the retinal neuronal and vascular degeneration is not clear. In this study we demonstrate that whenever a fraction of mouse or human adult bone marrow-derived stem cells (lineage-negative hematopoietic stem cells [Lin- HSCs]) containing endothelial precursors stabilizes and rescues retinal blood vessels that would ordinarily completely degenerate, a dramatic neurotrophic rescue effect is also observed. Retinal nuclear layers are preserved in 2 mouse models of retinal degeneration, $r d 1$ and $r d 10$, and detectable, albeit severely abnormal, electroretinogram recordings are observed in rescued mice at times when they are never observed in control-treated or untreated eyes. The normal mouse retina consists predominantly of rods, but the rescued cells after treatment with Lin- HSCs are nearly all cones. Microarray analysis of rescued retinas demonstrates significant upregulation of many antiapoptotic genes, including small heat shock proteins and transcription factors. These results suggest a new paradigm for thinking about the relationship between vasculature and associated retinal neuronal tissue as well as a potential treatment for delaying the progression of vision loss associated with retinal degeneration regardless of the underlying genetic defect.
\end{abstract}

\section{Introduction}

Inherited degenerations of the retina affect as many as 1 in 3,500 individuals and are characterized by progressive night blindness, visual field loss, optic nerve atrophy, arteriolar attenuation, altered vascular permeability, and central loss of vision often progressing to complete blindness (1). Molecular genetic analysis of these diseases has identified mutations in over 110 different genes accounting for only a relatively small percentage of the known affected individuals $(2,3)$; many of these mutations are associated with enzymatic and structural components of the phototransduction machinery including rhodopsin (4), cGMP phosphodiesterase (5), peripherin (6), and RPE65 (7). Despite these observations, there are still no effective treatments to slow or reverse the progression of these diseases. Recent advances in gene therapy have led to successful reversal of the retinal degeneration, slow $(r d s)(8)$ and retinal degeneration $(r d)$ (9) phenotypes in mice and the RPE65 phenotype in dogs (10) when the WT transgene is delivered to photoreceptors (PRs) or the reti-

Nonstandard abbreviations used: CIV, collagen IV; EPC, endothelial progenitor cell; ERG, electroretinogram; hBM, human bone marrow; hLin- HSC, human lineage-negative hematopoietic stem cell; HSC, hematopoietic stem cell; INL, inner nuclear layer; $\mathrm{Lin}^{-}$, lineage negative; $\mathrm{Lin}^{+}$, lineage positive; ONL, outer nuclear layer; $\mathrm{P}$, postnatal day; $\mathrm{PR}$, photoreceptor; $r d$, retinal degeneration; $r d 1$, retinal degeneration 1 mutation; $r d s$, retinal degeneration, slow; RP, retinitis pigmentosa.

Conflict of interest: The authors have declared that no conflict of interest exists.

Citation for this article: J. Clin. Invest. 114:765-774 (2004)

doi:10.1172/JCI200421686 nal pigmented epithelium in animals with a specific mutation. The potential use of calcium channel blockers (11), trophic factors (12), and dietary supplements (13) has also been explored in recent studies. Most inherited human retinal degenerations specifically affect rod PRs, but there is concomitant loss of cones, the principal cellular component of the macula, the region of the retina in humans that is responsible for central, fine visual acuity. Cone-specific survival factors have been described recently (14) and may facilitate cone survival in mouse models of retinal degeneration.

Adult bone marrow is known to contain a population of hematopoietic stem cells (HSCs) that can be divided into lineage positive $\left(\mathrm{Lin}^{+}\right)$and lineage negative $\left(\mathrm{Lin}^{-}\right)$subpopulations with regard to their potential to differentiate into formed elements of the blood. The Lin' population contains a variety of progenitor cells including those capable of becoming vascular endothelial cells (15). These endothelial progenitor cells (EPCs) mobilize from the bone marrow in response to a variety of signaling molecules $(16,17)$ and can target sites of angiogenesis in ischemic peripheral vasculature (15), myocardium (18), or induced ocular injury (19). We have reported that Lin $^{-}$HSCs injected directly into the eye can target activated astrocytes and participate in normal developmental angiogenesis in neonatal mice or injury-induced neovascularization in the adult (20). We now report that adult bone marrow-derived Lin ${ }^{-}$HSCs, when injected intravitreally up to 2 weeks postnatally, can completely prevent retinal vascular degeneration ordinarily observed in the $r d 1$ or $r d 10$ mouse models 


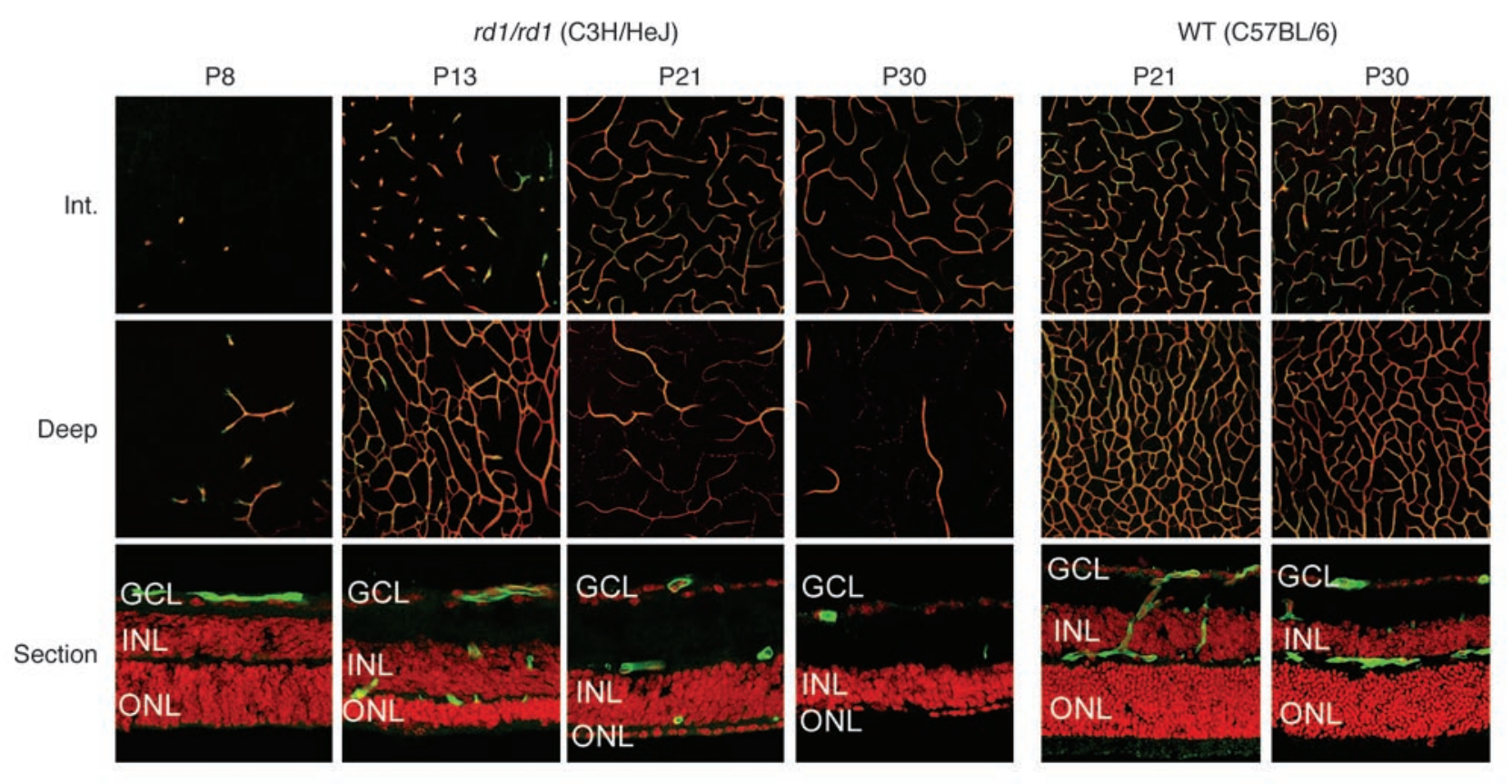

Figure 1

Retinal vasculature and neural cell changes in rd1/rd1 (C3H/HeJ, left panels) or WT mice (C57BL/6, right panels). Retinal vasculature of intermediate (top row) or deep (middle row) vascular plexuses in whole-mounted retinas (red: CIV; green: CD31) and sections (bottom row; red: DAPI; green: CD31) of the same retinas are shown. GCL, ganglion cell layer; Int., intermediate plexus; deep, deep plexus.

of retinal degeneration, and this vascular rescue correlates with neuronal rescue. The inner nuclear layer (INL) remains nearly normal, and the outer nuclear layer (ONL) containing PRs is significantly preserved with the rescued cells being predominantly cones. Detectable, albeit severely abnormal, electroretinogram (ERG) recordings are observed in rescued mice at times when they are never observed in control-treated, or untreated, $r d / r d$ eyes. This rescue effect is also observed when human bone marrow-derived (hBM-derived) Lin ${ }^{-}$HSCs are used to treat SCID mice with retinal degeneration. Large-scale genomic analysis of rescued and nonrescued eyes revealed significant upregulation of antiapoptotic genes. These findings demonstrate that this newly described neurotrophic effect correlates with preservation of the vasculature and suggest that autologous bone marrow-derived EPCs may be useful in the treatment of currently untreatable blinding disease.

\section{Results}

The $r d 1 / r d 1$ mouse retinas exhibit profound vascular and neuronal degeneration. Normal postnatal retinal vascular and neuronal development in mice has been well described and is analogous to changes observed in the third-trimester human fetus (21). Mice homozygous for the $r d 1$ gene share many characteristics of human retinal degeneration (11) and exhibit rapid PR loss accompanied by severe vascular atrophy as the result of a mutation in the gene encoding PR cGMP phosphodiesterase (5). To examine the vasculature during retinal development and its subsequent degeneration, we used Ab's against collagen IV (CIV), an ECM protein of mature vasculature, and CD31 (PECAM-1), a marker for endothelial cells (Figure 1). Retinas of $r d 1 / r d 1$ (C3H/HeJ) developed normally until approximately postnatal day 8 (P8), when degeneration of the PR containing the ONL begins. The ONL rapidly degenerates, and cells die by apoptosis (22), such that only a single layer of nuclei remains by P20 (23). Double staining of the whole-mounted retinas with Ab's to both CIV and CD31 revealed details of the vascular degeneration in $r d 1 / r d 1$ mice similar to that described by others (24). The primary and deep retinal vascular layers appear to develop normally through P12, after which there is a rapid loss of endothelial cells as evidenced by the absence of CD31 staining; CD31-positive endothelial cells are present in a normal distribution through $\mathrm{P} 12$ but rapidly disappear after that. Interestingly, CIV-positive staining remains present throughout the time points examined, suggesting that the vessels and associated ECM form normally, but that only the matrix remains after P13, by which time no CD31-positive cells are observed. (Figure 1, middle panels). The intermediate vascular plexus also degenerates after P21, but the progression is slower than that observed in the deep plexus (Figure 1, upper panel). Retinal vascular and neural cell layers of a normal mouse are shown for comparison with the $r d 1 / r d 1$ mouse (right panels, Figure 1).

Neuroprotective effect of bone marrow-derived Lin $^{-}$HSCs in $\mathrm{rd} 1 / \mathrm{rd} 1$ mice. We have reported that intravitreally injected Lin $^{-}$HSCs incorporate into endogenous retinal vasculature in all three vascular plexuses and prevent the vessels from degenerating (20). Interestingly, the injected cells are never observed in the ONL; these cells either incorporate into the forming retinal vessels or are observed in close proximity to these vessels. To investigate whether Lin- HSCs could also slow or prevent the degeneration of neuronal cells in mouse eyes with retinal degeneration we injected $\mathrm{Lin}^{-} \mathrm{HSCs}$ (from C3H/HeJ) intravitreally into C $3 \mathrm{H} / \mathrm{HeJ}(r d 1 / r d 1)$ mouse eyes at P6, just prior to the onset of degeneration. By P30, control cell-injected $\left(\mathrm{CD} 31^{-}\right)$eyes exhibited the typical $r d 1 / r d 1$ phenotype; nearly complete degeneration of the deep vascular plexus, and ONL was observed in every retina examined. Eyes injected with Lin- HSCs maintained normal-appearing interme- 


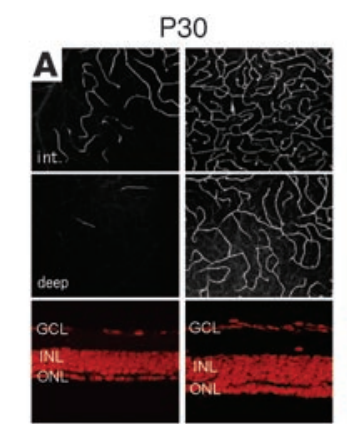

Control
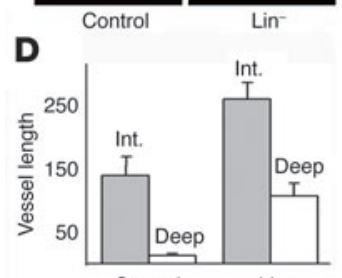

E
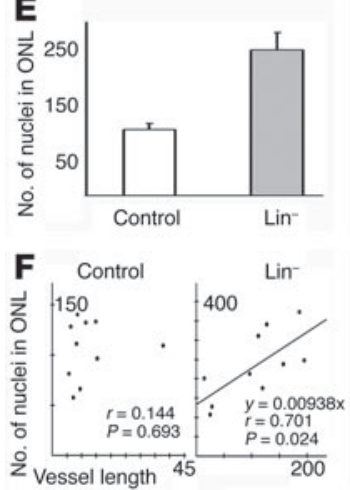

P60
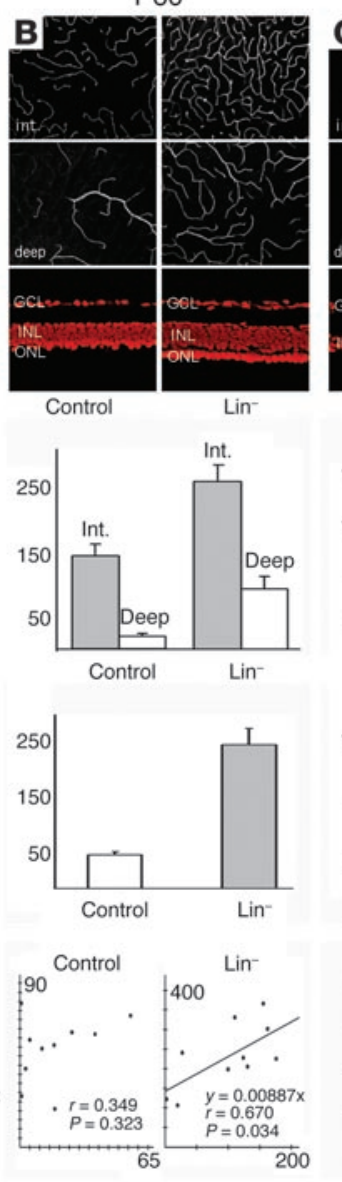

P180
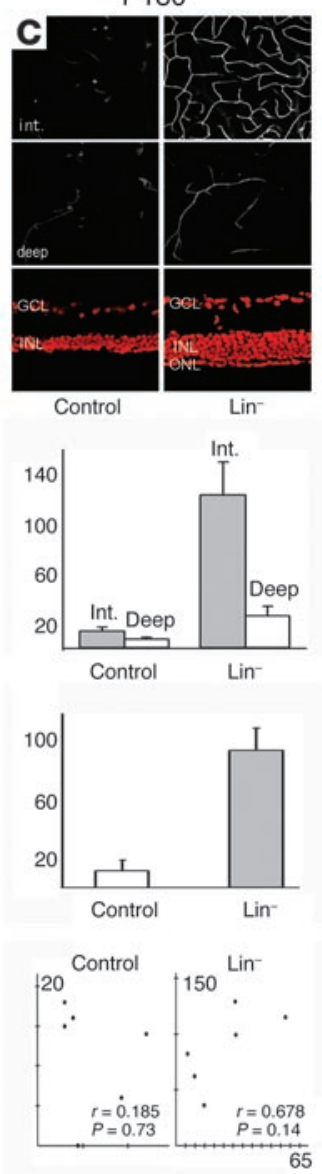

\section{Figure 2}

$\mathrm{Lin}^{-} \mathrm{HSC}$ injection rescues the degeneration of vascular and neural cells in $r d 1 /$ rd1 mice. (A-C) Retinal vasculature of intermediate or deep plexus and cross sections of Lin- HSC-injected eye (Lin-, right panels in each part) and contralateral control cell-injected (CD31-) eye (left panels in each part) at P30 (A), P60 (B), and P180 (C). (D) The average total length of vasculature $( \pm$ SEM) in Lin $^{-}$HSC-injected or control cell-injected $\left(C D 31^{-}\right)$retinas at P30 (left, $n=10$ ), P60 (middle, $n=10$ ), and P180 (right, $n=6$ ). Data of intermediate and deep vascular plexus are shown separately ( $y$ axis: relative length of vasculature). (E) The average numbers of cell nuclei in the ONL at P30 (left, $n=10$ ), P60 (middle, $n=10$ ), or P180 (right, $n=6$ ) of control cell-injected $\left(\mathrm{CD}^{-} 1^{-}\right)$or $\mathrm{Lin}^{-} \mathrm{HSC}-$ injected retinas $(y$ axis: relative number of cell nuclei in the $\mathrm{ONL}$ ). (F) Simple linear correlations between the length of vasculature ( $x$ axis) and the number of cell nuclei in the ONL ( $y$ axis) at P30 (left), P60 (middle), and P180 (right) of Lin- HSC-injected or control cell-injected retinas. diate and deep vascular plexuses and, surprisingly, significantly more cells in the INL and ONL than control cell-injected eyes (Figure 2A). This rescue effect of Lin- HSCs can be observed at 2 months (Figure 2B) and for as long as 6 months after injection (Figure 2C). The effect is observed by injecting Lin- HSCs up to 16 days postnatally; injection after this time did not produce significant rescue (data not shown). Differences in the vasculature of the intermediate and deep plexuses, as well as the neuronal cell-containing INL and ONL, were significant at all time points measured when rescued and nonrescued eyes were compared (Figure $2, \mathrm{~A}-\mathrm{C})$. This effect was quantified by measuring the total length of the vasculature (Figure 2D) and counting the number of DAPIpositive cell nuclei observed in the ONL (Figure 2E).

To determine whether there is a relationship between the length of the vasculature and the number of ONL cells, simple linear-regression analysis was applied to the data at all time points. We consistent-

\section{Figure 3}

Retinal function is rescued by Lin- HSC injection. ERG recordings were used to measure the function of Lin- HSC-injected or control cell-injected $\left(\mathrm{CD} 31^{-}\right)$retinas. ( $\mathbf{A}$ and $\left.\mathbf{B}\right)$ Representative cases of rescued and nonrescued retinas 2 months after injection. Retinal section of Lin- HSC-injected right eye (A) and CD31- control cell-injected left eye (B) of the same animal are shown (green: CD31-stained vasculature; red: DAPI-stained nuclei). (C) ERG responses from the same eyes shown in $\mathbf{A}$ and $\mathbf{B}$.

ly observed a statistically significant correlation between vascular rescue and neuronal (e.g., ONL thickness) rescue at P30 $(P<0.024)$ and P60 $(P<0.034)$ in the Lin ${ }^{-}$HSC-injected eyes (Figure $\left.2 \mathrm{~F}\right)$. The correlation remains high, although not statistically significant $(P<0.14)$, at P180 when comparing Lin $^{-}$HSC-injected retinas to control cell-injected retinas (Figure 2F). In contrast, control cell-injected retinas showed no significant correlation between the preservation of vasculature and ONL at any time point (Figure $2 \mathrm{~F}$ ). These data demonstrate that intravitreal injection of Lin' ${ }^{-}$HSCs results in concomitant retinal vascular and neuronal rescue in retinas of $r d 1 / r d 1$ mice. Injected cells were never observed in the ONL or any place other than within, or in close proximity to, retinal blood vessels.

A
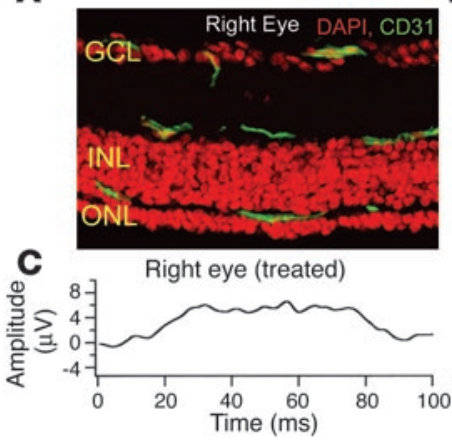

B
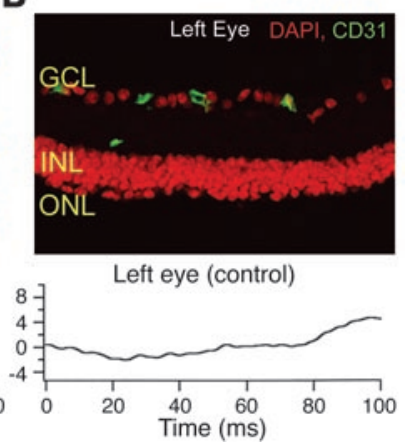


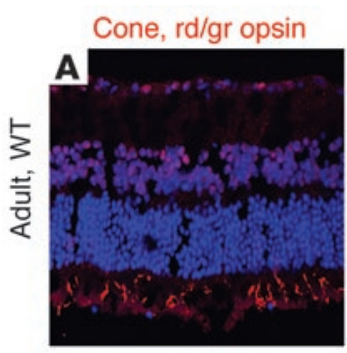

Noninjected

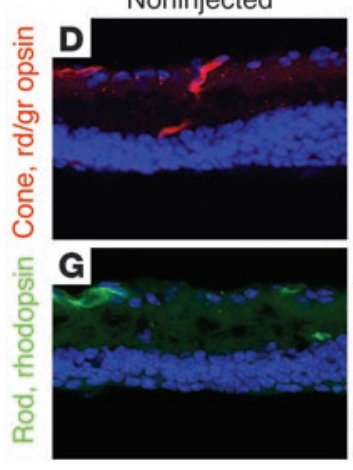

Rod, rhodopsin

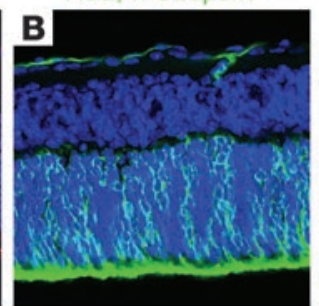

CD31- HSC-injected

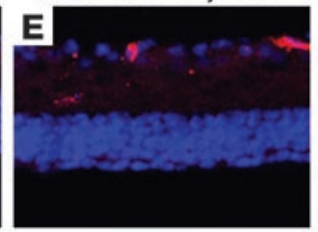

E

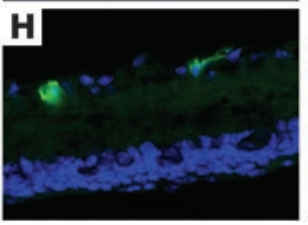

Preimmune IgG

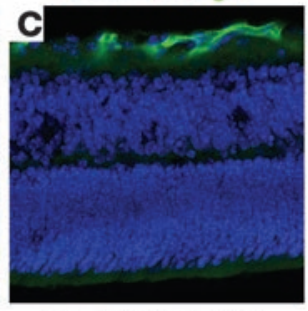

Lin- HSC-injected

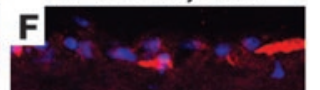

Figure 4

Rescued PRs in rd1 ONL following intravitreal injection of Lin- HSCs are predominantly cones. A small percentage of PRs in the WT mouse retina (upper images) are cones as evidenced by expression of red/green cone opsin (A), while most cells of the ONL are positive for rod-specific rhodopsin (B). Retinal vasculature autofluoresces with preimmune serum (C), but nuclear layers are completely negative for staining with rod- or cone-specific opsins. The $r d / r d$ mouse retinas (lower images) have a diminished INL and a nearly completely atrophic ONL, both negative for cone (D) or rod (G) opsin. Control, CD31- HSC-treated eyes are identical to noninjected $r d / r d$ retinas, without any staining for cone (E) or rod (H) opsin. Lin- HSC-treated contralateral eyes have a markedly reduced, but clearly present, ONL that is predominantly made up of cones as evidenced by positive immunoreactivity for red/green cone opsin (F). A small number of rods are also observed (I). rd, red; gr, green.

Functional rescue of $\mathrm{Lin}^{-} \mathrm{HSC}-$ injected $r d / r d$ retinas. To test whether Lin- HSC treatment could also induce functional rescue of vision in $r d 1 / r d 1$ mice, we performed ERGs on mice 2 months after injection of control or Lin ${ }^{-}$HSCs. Immunohistochemical and microscopic analyses (Figure 3, A and B) were done with each eye following ERG recordings to confirm that vascular and neuronal rescue had, in fact, occurred. Representative ERG recordings from treated rescued and control nonrescued eyes are shown in Figure 3C. Clearly, the signal from the rescued eye is severely abnormal. Consistent and detectable ERGs with amplitudes on the order of 8-10 $\mu \mathrm{V}$ were recordable from the $\mathrm{Lin}^{-}$HSC-treated eyes, however; in all cases the ERG from the control eye was nondetectable. While the amplitudes of the signals in rescued eyes were considerably lower than normal and their pattern atypical, they were consistently observed whenever there was histological rescue and are on the order of magnitude of those reported by other, gene-based, rescue studies. Overall, these results are suggestive of some degree of functional rescue in treated eyes.

Rescued $r d / r d$ retinal cell types are predominantly cones. To determine the cellular basis of the functional rescue, we analyzed rescued and nonrescued retinas immunohistochemically with Ab's specific for rod or cone opsin. The same eyes used for the ERG recordings presented in Figure 3 were analyzed for rod or cone opsin. In WT mouse retinas, less than $5 \%$ of PRs present are cones (25), and the immunohistochemical staining patterns we observed with red/ green cone opsin (Figure 4A) or rod rhodopsin (Figure 4B) are consistent with this. When WT retinas are stained with preimmune IgG, no staining is observed anywhere in the neurosensory retinas other than autofluorescence of the blood vessels (Figure 4C). Two months after birth, retinas of noninjected $r d / r d$ mice have an essentially atrophic ONL that does not exhibit any staining with Ab's to red/green cone opsin (Figure 4D) or rhodopsin (Figure 4G). Eyes injected with control CD31- HSCs also do not stain positively for the presence of either cone (Figure 4E) or rod (Figure $4 \mathrm{H}$ ) opsin. In contrast, contralateral eyes injected with Lin $^{-}$HSC have three to six rows of nuclei in a preserved ONL; most of these cells are positive for cone opsin (Figure 4F), with approximately $1-3 \%$ positive for rod opsin (Figure $4 \mathrm{I}$ ). Remarkably, this is nearly the reverse of what is ordinarily observed in the normal mouse retina, which is rod dominated (26). These data suggest that the injection of $\mathrm{Lin}^{-}$HSCs preserves cones for extended periods of time during which they would ordinarily degenerate.

The hBM-derived Lin $^{-}$HSCs also rescue degenerating retinas. We have also examined whether there is a population of cells in hBM that behave similarly to mouse Lin $^{-}$HSCs. Bone marrow was collected from human donors and the $\mathrm{Lin}^{+} \mathrm{HSCs}$ were depleted, producing a population of human $\mathrm{Lin}^{-} \mathrm{HSCs}$ (hLin- HSCs). These cells were labeled ex vivo with fluorescent dye and injected into C3SnSmn. CB17-Prkdc ${ }^{S C I D}$ mouse eyes. The injected hLin ${ }^{-}$HSCs migrated to and targeted sites of retinal angiogenesis in a fashion identical to that observed when mouse Lin- HSCs were injected (Figure 5A) (20). In addition to the vascular targeting, the human stem cells also provided a robust rescue effect on both the vascular and neuronal cell layers of the $r d 1 / r d 1$ mice (Figure 5, B and C). This observation confirms the presence of cells in hBM that target retinal vasculature and can prevent its degeneration in mice with inherited retinal degeneration.

Lin $^{-}$HSCs have vasculotrophic and neurotrophic effects in the rd10/ $r d 10$ mouse. While the $r d 1 / r d 1$ mouse is the most widely used and best-characterized model for retinal degeneration (26), the degeneration is very rapid and in this regard differs from the usual, slower time course observed in the human disease. In this strain, PR cell degeneration begins around P8, a time when the retinal vasculature is still rapidly expanding (Figure 1). Subsequent degeneration of the deep retinal vasculature occurs even while the intermediate plexus is still forming, and thus the retinas of $r d 1 /$ rd1 mice never completely develop, unlike that observed in most humans with this disease. To investigate whether HSC-mediated vascular rescue could prevent retinal neuronal degeneration that occurs after the neurosensory retina and its corresponding vasculature is largely developed, we decided to use another model for retinal degeneration that has a slower time course of degenera- 
A

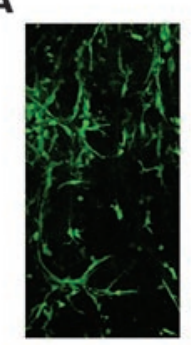

D
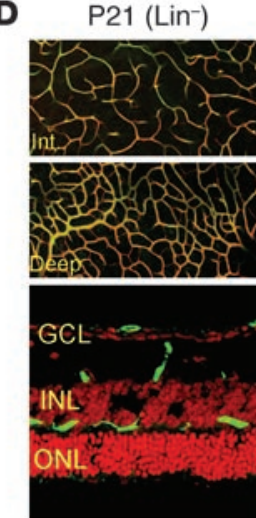

H P21 (Control)
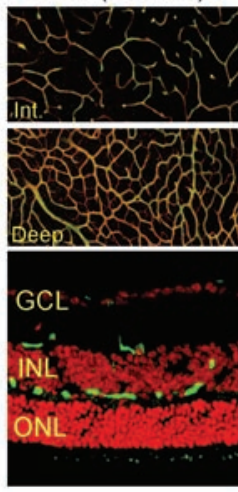

B
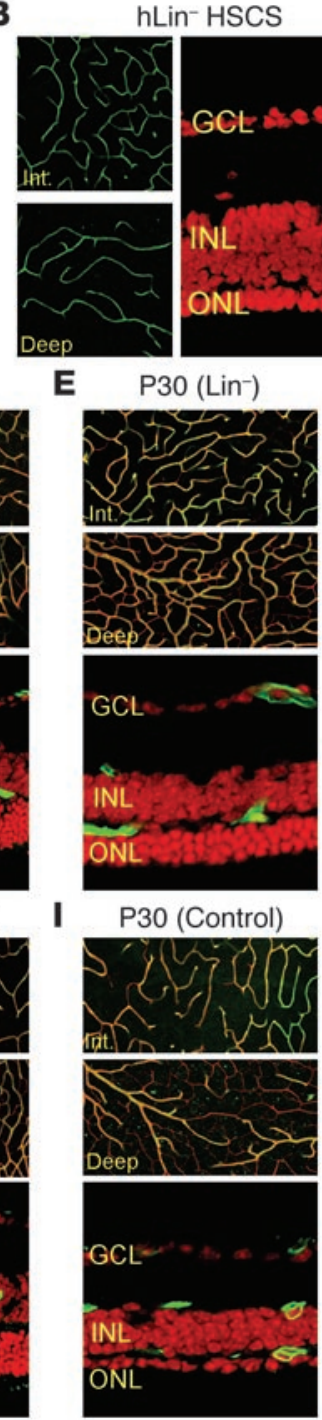

C

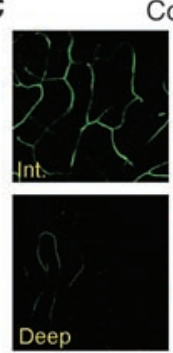

$\mathbf{F}$
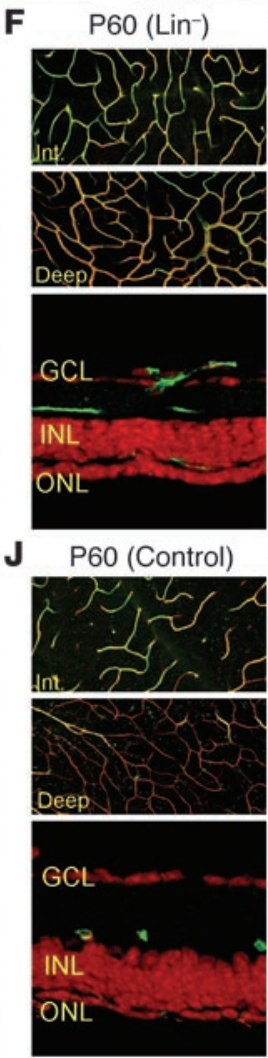

Control

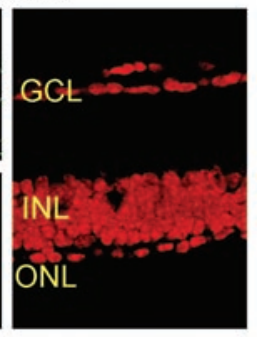

G
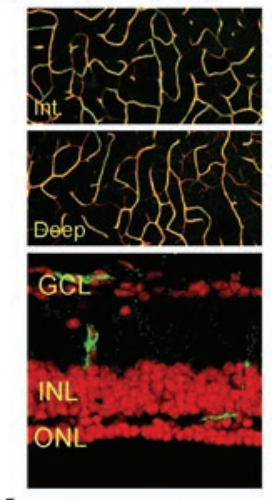

K P105 (Control)
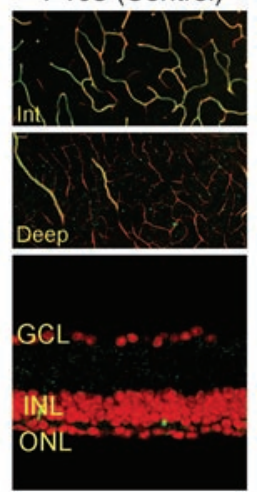

\section{Figure 5}

A population of $h B M$ cells can rescue degenerating retinas in the $r d 1$ mouse $(\mathbf{A}-\mathbf{C})$, and the rescue is also observed using mouse bone marrow-derived Lin- HSCs in another model of retinal degeneration, $r d 10$ (D-K). (A) $\mathrm{hLin}-\mathrm{HSC}$ s labeled with green dye targeted to developing vasculature after intravitreal injection into C3SnSmn.CB17-Prkdc SCID mice. (B and $\mathbf{C}$ ) Retinal vasculature (upper-left panels: intermediate plexus; lower-left panels, deep plexus) and neural cells (right panels) in $\mathrm{hLin}^{-}$ HSC-injected eye (B) or contralateral control eye (C) 1.5 months after injection. (D-K) Rescue of $r d 10$ mice by Lin- HSCs (injected at P6). Representative retinas at P21 (D: LinHSCs; H: control cells), P30 (E: Lin- HSCs; I: control cells); P60 (F: Lin- HSCs; J: control cells), and P105 (G: Lin- HSCs; K: control cells) are shown (treated and control eyes are from the same animal at each time point). Retinal vasculature (upper image in each part is the intermediate plexus; the middle image in each part is the deep plexus) was stained with CD31 (green) and CIV (red). The lower image in each part shows a cross section made from the same retina (red: DAPI; green: CD31). tion and, thus, may more closely resemble the human condition. In the $r d 10$ mouse, PR cell degeneration begins around P21 (26), and shortly after this, vascular degeneration begins. Since normal neurosensory retinal development is largely complete by $\mathrm{P} 21$, the degeneration is observed to start after the retina has completed differentiation and in this way is more analogous to human retinal degenerations. We injected Lin ${ }^{-}$HSCs or control cells from rd10 mice into $\mathrm{P} 6$ eyes and evaluated the retinas at varying time points. At P21 the retinas from both Lin $^{-}$HSC- and control cellinjected eyes appeared normal, with complete development of all vascular layers and normal development of the INL and ONL (Figure 5, D and $\mathrm{H}$ ). At approximately P21, the retinal degeneration begins and progresses with age. By P30, the control cell-injected retinas exhibited severe vascular and neuronal degeneration (Figure 5I), while the Lin- HSC-injected retinas maintained nearly normal vascular layers and PR cells (Figure 5E). The difference between rescued and nonrescued eyes was most pronounced at P30 (compare Figure 5E with Figure 5I), but is also observed at later time points (compare Figure 5, F and $\mathrm{G}$ with $\mathrm{J}$ and $\mathrm{K}$ ). In the control-treated eyes, the progression of vascular degeneration was very clearly observed by immunohistochemical staining for CD31 and CIV (Figure 5, I-K); these eyes were nearly completely negative for CD31 whereas CIV-positive vascular "tracks" remained evident, indicating that vascular regression, rather than incomplete vascular formation, had occurred. In contrast, Lin ${ }^{-}$HSC-injected eyes had both CD31 and CIV-positive vessels that appeared very similar to normal, WT eyes (compare Figure 5E with Figure 5I and with WT vasculature in Figure 1, rightmost panel).

Gene expression analysis of $r d / r d$ mouse retinas after $\mathrm{Lin}^{-} \mathrm{HSC}$ treatment. To begin to identify putative mediators of the neurotrophic rescue effect in mice with retinal degeneration, we used large-scale genomics (microarray analysis) to analyze rescued and nonrescued retinas. Gene expression in $r d 1 / r d 1$ mouse retinas treated with Lin- HSCs was compared with uninjected retinas as well as retinas injected with control cells $\left(\mathrm{CD} 31^{-}\right)$. These experiments were each done in triplicate. To be considered present, genes were required to have expression levels at least twofold higher than background levels in all three triplicates. Genes that were upregulated threefold in $\mathrm{Lin}^{-} \mathrm{HSC}$-protected retinas compared with control cell-injected and noninjected $r d / r d$ mouse retinas are shown in Table 1. Coefficient of variance levels were calculated for the expressed genes by dividing the SD by the mean expression 
Table 1

Gene expression analysis

\begin{tabular}{|c|c|c|c|c|c|c|c|c|}
\hline \multirow[b]{2}{*}{ Common name } & \multicolumn{3}{|c|}{ (Normalized data) } & \multirow[b]{2}{*}{ Genbank no. } & \multirow[b]{2}{*}{ Keywords } & \multicolumn{3}{|c|}{$P$ value } \\
\hline & Lin- $^{-}$ & CD31- & $\mathrm{NI} r d / r d$ & & & $\operatorname{Lin}^{-}$ & CD31- & $\mathrm{NI} r d / r d$ \\
\hline \multicolumn{9}{|c|}{ Genes whose expression is increased 3-fold in Lin- HSC-injected retinas } \\
\hline Tgtp & 11.86 & 0.53 & 0.66 & L38444 & T cell-specific protein & $0.015(P)$ & $0.162(A)$ & $0.181(A)$ \\
\hline$H-2 D 4(q)$ & 7.09 & 0.92 & 0.69 & X52914 & Transplantation antigen & $0.016(P)$ & $0.035(A)$ & $0.054(A)$ \\
\hline$H 2-K 2 ; H-2 K 2$ & 4.51 & 0.70 & 0.55 & M27134 & Cell surface glycoprotein & $<0.001(\mathrm{P})$ & $0.040(P)$ & $0.120(A)$ \\
\hline Lzp-s & 6.51 & 0.65 & 0.99 & X51547 & Lysozyme; lysozyme P & $0.015(P)$ & $0.011(\mathrm{~A})$ & $0.003(\mathrm{~A})$ \\
\hline Kcnj5 & 4.50 & 0.85 & 0.72 & U33631 & G protein-gated $\mathrm{K}^{+}$channel & $0.037(\mathrm{P})$ & $0.022(A)$ & $0.084(A)$ \\
\hline Scya8 & 5.19 & 0.47 & 1.00 & AB023418 & MCP-2 precursor & $0.004(\mathrm{P})$ & $0.060(A)$ & $0.013(\mathrm{~A})$ \\
\hline Ly6a & 4.02 & 0.96 & 0.79 & X04653 & Ly-6 alloantigen & $0.014(P)$ & $0.014(\mathrm{P})$ & $0.021(\mathrm{~A})$ \\
\hline Pip5k1c & 3.40 & 0.94 & 0.78 & AB006916 & Phosphatidylinositol kinase & $0.012(P)$ & $0.013(\mathrm{~A})$ & $0.035(A)$ \\
\hline$M A D$ & 3.76 & 0.56 & 0.89 & X83106 & MAX dimerization protein & $0.013(P)$ & $0.080(A)$ & $0.078(\mathrm{~A})$ \\
\hline Cxadr & 3.98 & 0.81 & 1.00 & U90715 & Coxsackie-adenovirus receptor & $0.011(P)$ & $0.011(P)$ & $<0.001(\mathrm{P})$ \\
\hline $\operatorname{lsg} 15$ & 2.22 & 0.64 & 0.45 & X56602 & INF-inducible protein & $0.010(P)$ & $0.086(A)$ & $0.129(\mathrm{~A})$ \\
\hline YY1 & 2.97 & 0.85 & 0.87 & M74590 & $\delta$-transcription factor & $0.039(\mathrm{P})$ & $0.040(\mathrm{~A})$ & $0.006(A)$ \\
\hline Psmb9 & 3.29 & 0.49 & 0.97 & D44456 & Polypeptide complex subunit 2 & $0.015(P)$ & $<0.001(A)$ & $0.043(A)$ \\
\hline \multicolumn{9}{|c|}{ Crystallin genes whose expression is increased 3-fold in Lin- HSC-injected retinas } \\
\hline Crybb2 & 8.73 & 0.55 & 0.83 & M60559 & $\beta$ B2 crystallin & $0.008(P)$ & $0.047(\mathrm{~A})$ & $0.085(A)$ \\
\hline Cryaa & 4.00 & 0.57 & 1.00 & J00376 & $\alpha A$-crystallin & $0.002(P)$ & $0.015(A)$ & $0.003(A)$ \\
\hline CrygD & 2.09 & 0.74 & 0.97 & AJ224342 & $\gamma \mathrm{D}$-crystallin & $0.017(\mathrm{P})$ & $<0.001(\mathrm{P})$ & $0.033(P)$ \\
\hline Cryba1 & 6.52 & 0.93 & 0.60 & AJ239052 & $\beta A 3 / A 1-c r y s t a l l i n$ & $0.007(P)$ & $0.036(P)$ & $0.055(\mathrm{~A})$ \\
\hline Crygs & 2.89 & 0.97 & 0.85 & AF032995 & $\gamma \mathrm{S}$-crystallin & $0.007(\mathrm{P})$ & $0.017(P)$ & $0.006(P)$ \\
\hline CrygC & 5.07 & 1.00 & 0.83 & Z22574 & $\gamma \mathrm{C}$-crystallin & $0.029(\mathrm{P})$ & $<0.001(\mathrm{~A})$ & $0.004(\mathrm{~A})$ \\
\hline CrygF & 1.94 & 1.00 & 0.69 & AJ224343 & $\gamma$ F-crystallin & $0.010(P)$ & $0.007(\mathrm{P})$ & $<0.001(A)$ \\
\hline
\end{tabular}

A, absent, P, present. NI, noninjected.

level of each copy RNA (cRNA) replicate. In addition, the correlation between expression levels and noise variance was calculated by correlating the mean $[\ln ($ expression $)]$ and $\mathrm{SD}[\ln ($ expression $)]$. This demonstrated a correlation between gene-expression levels and SDs for each gene, allowing us to determine background levels and reliable expression-level thresholds. As a whole, our data fell well within acceptable limits (27), and individually discussed genes fell above these critical expression levels. Paired $t$ test values for the discussed genes are also presented. In each case, $P$ values are reasonable (near or below 0.05 ), demonstrating similarities between replicates and probable significant differences between the different test groups. Many of the significantly upregulated genes, including MAD and Ying Yang-1 (28), encode proteins with functions involving the protection of cells from apoptosis. A number of crystallin genes, which have sequence homology and functions similar to known heat shock proteins involving protection of cells from stress, were also upregulated by Lin ${ }^{-}$HSC treatment. Expression of $\alpha$-crystallin was localized to the ONL by immunohistochemical analysis (Figure 6).

To identify factors directly expressed by the HSCs themselves, we hybridized mRNA from $r d 1 / r d 1$ mouse retinas rescued with hBM-derived HSCs to human-specific microarray chips. After stringent analysis, a number of genes were found whose mRNA expression was human specific, above background, and significantly higher in the human HSC-rescued retinas compared with the mouse HSC-rescued and human control cell-injected nonrescued retinas (Table 2). CD6, a cell adhesion molecule expressed at the surface of primitive and newly differentiated CD $34^{+}$hematopoietic stem cells (29), and IFNo-13, another gene expressed by hematopoietic stem cells (30), were both found by our microarray bioinformatics, helping to validate the experimental protocol. In addition, several growth factors and neurotrophic factors were expressed above background by human HSCs in rescued mouse retina samples (Table 2).

\section{Discussion}

We have demonstrated that adult bone marrow-derived Lin- HSCs exert profound vasculotrophic and neurotrophic effects when injected intravitreally into mice with retinal degenerative disease. This rescue effect persists for up to 6 months after treatment and is most efficacious when the Lin- HSCs are injected prior to complete retinal degeneration (up to 16 days after birth in mice that ordinarily exhibit practically complete ONL degeneration by 30 days postnatally). This rescue is observed in two mouse models of retinal degeneration and, remarkably, can be accomplished with adult hBM-derived HSCs when the recipient is an immunodeficient rodent with retinal degeneration (e.g., the SCID mouse) or
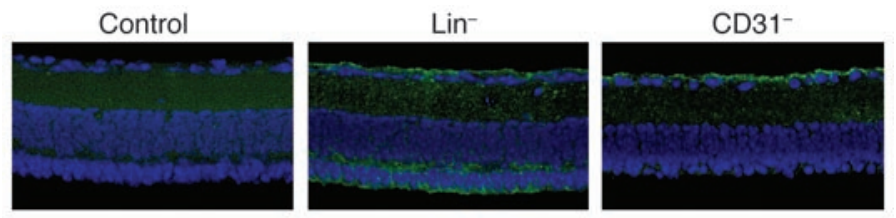

Figure 6

Green: crystallin $\alpha$ A; blue: DAPI

Crystallin $\alpha \mathrm{A}$ is upregulated in rescued ONL cells after treatment with LinHSCs but not in contralateral eyes treated with control cells. Left: IgG control in rescued retina; middle: crystallin $\alpha \mathrm{A}$ in rescued retina; right: crystallin $\alpha A$ in nonrescued retina. 
Table 2

Gene expression analysis

\begin{tabular}{|c|c|c|c|c|c|c|c|c|}
\hline \multirow[b]{2}{*}{ Common Name } & \multicolumn{3}{|c|}{ (Raw data) } & \multirow[b]{2}{*}{ Genbank no. } & \multirow[b]{2}{*}{ Keywords } & \multicolumn{3}{|c|}{$P$ value } \\
\hline & MR & HR & HNR & & & $\operatorname{Lin}^{-}$ & CD31- & $\mathrm{NI} r d / r d$ \\
\hline \multicolumn{9}{|c|}{ Genes upregulated at least twofold in human stem cell-rescued retinas } \\
\hline EST & 397 & 1885 & 489 & AK024177 & EST & 0.024 & 0.007 & 0.006 \\
\hline CD6 & 966 & 2854 & 840 & NM_006725 & CD6 antigen & 0.011 & 0.065 & $<0.001$ \\
\hline IFNa13 & 241 & 606 & 220 & NM_006900 & IFN $\alpha-13$ & 0.010 & 0.063 & 0.013 \\
\hline TRAG3 & 269 & 615 & 229 & NM_004909 & Taxol resistance-associated gene 3 & 0.028 & 0.052 & 0.001 \\
\hline ELA2 & 229 & 545 & 187 & NM_001972 & Elastase 2, neutrophil & 0.003 & 0.070 & 0.013 \\
\hline PP32D & 179 & 411 & 146 & NM_012404 & Acidic nuclear phosphoprotein 32D & 0.003 & 0.067 & 0.002 \\
\hline Otoferlin & 232 & 527 & 239 & NM_004802 & Otoferlin & 0.002 & 0.050 & 0.008 \\
\hline Acyl oxidase & 175 & 391 & 164 & NM_004035 & Acyl-coenzyme A oxidase & $<0.001$ & 0.064 & 0.001 \\
\hline HS6ST1 & 204 & 455 & 210 & NM_004807 & Heparan sulfate sulfotransferase & 0.010 & 0.063 & 0.004 \\
\hline ACCN3 & 187 & 413 & 181 & NM_004769 & Amiloride-sensitive cation channel & 0.007 & 0.070 & 0.002 \\
\hline \multicolumn{9}{|c|}{ Neurotrophic or growth factor genes upregulated in human HSC-rescued retinas } \\
\hline IGF2 & 743 & 1382 & 839 & NM_016412 & IGF-2 & $<0.001$ & 0.041 & 0.007 \\
\hline SCGF & 474 & 547 & 402 & NM_002975 & Stem cell growth factor & 0.003 & 0.002 & 0.009 \\
\hline$T G F, \beta 1$ & 841 & 1012 & 819 & NM_000660 & TGF, $\beta 1$ & 0.003 & 0.005 & 0.002 \\
\hline$T G F, \beta 1$ & 144 & 252 & 122 & NM_0006660 & (Second probe along TGF- $\beta$ mRNA) & 0.006 & 0.059 & 0.017 \\
\hline EGFR S8 & 499 & 593 & 491 & Al343292 & EGF receptor pathway substrate 8-related protein 1 & 0.010 & 0.006 & 0.008 \\
\hline FGF-6 & 524 & 613 & 526 & NM_020996 & FGF-6 & 0.004 & 0.010 & 0.005 \\
\hline$E S T(I G F B P ?)$ & 927 & 991 & 895 & AW338791 & Similarities to IGF-binding proteins & 0.006 & 0.006 & 0.003 \\
\hline FGF-12 & 471 & 523 & 507 & AL119322 & FGF-12 & 0.006 & 0.001 & $<0.001$ \\
\hline EGF-like & 991 & 1092 & 1088 & M60278 & Diphtheria toxin receptor, EGF-like & 0.004 & 0.004 & 0.003 \\
\hline FGF-21 & 592 & 615 & 590 & NM_019113 & FGF-21 & 0.005 & 0.001 & 0.004 \\
\hline
\end{tabular}

MR, mouse rescued; HR, human rescued; HNR, human nonrescued.

when the donor is itself a mouse with retinal degeneration. PRs in the normal mouse retina are predominantly rods, but the ONL observed after rescue with Lin- HSCs contains predominantly cones. While several recent reports have described a partial phenotypic rescue in mice or dogs with retinal degeneration after viralbased gene therapy with the WT gene (8-10), this is the first report of a generic cell-based therapeutic effect correlating with vascular rescue. When attempting to treat a group of diseases (e.g., retinitis pigmentosa, or RP) that can be caused by mutations in over 100 known genes, such an approach is potentially more practical than creating individual gene therapies to treat each mutation. Most inherited human retinal degenerations occur as a result of primary rod-specific defects, and loss of the cones is believed to be secondary to rod dysfunction, perhaps related to the loss of some trophic factor expressed by rods (14). Our observation that cone survival in the face of rod/retinal degeneration is facilitated by Lin $^{-}$HSCs suggests that it may be possible to better preserve the cone-dominated human macula in diseases such as RP.

The precise molecular basis of the neurotrophic rescue effect remains unknown but is observed only when there is concomitant vascular stabilization/rescue. The presence of injected stem cells, per se, is not sufficient to generate a neurotrophic rescue, and the clear absence of stem cell-derived neurons in the ONL rules out the possibility that the injected cells are transforming into PRs. Data obtained by microarray gene expression analysis demonstrated a significant upregulation of genes known to have antiapoptotic effects. Since most neuronal death observed in retinal degenerations is by apoptosis, such protection may be of great therapeutic benefit in prolonging the life of PRs and other neurons critical to visual function in these diseases. C-myc is a tran- scription factor that participates in apoptosis (31) by upregulating various downstream apoptosis-inducing factors. C-myc expression was increased 4.5-fold in $r d / r d$ mice over WT (data not shown), indicating potential involvement in the PR degeneration observed in the $r d 1 / r d 1$ mouse. Mad1 and $Y Y-1$, two genes dramatically upregulated in $\mathrm{Lin}^{-}$-protected retinas (Table 1), are known to suppress the activity of c-myc, thus inhibiting c-myc-induced apoptosis $(28,32)$. Overexpression of Mad1 has also been shown to suppress Fas-induced activation of caspase-8 (32), another critical component of the apoptotic pathway. Upregulation of these two molecules may play a role in protection of the retina from vascular and neural degeneration by preventing the initiation of apoptosis that normally leads to degeneration in $r d / r d$ mice.

Another set of genes that were greatly upregulated in $\mathrm{Lin}^{-}$ HSC-protected retinas includes members of the crystallin family (Table 1B). Similar to heat shock and other stress-induced proteins, crystallins may be activated by retinal stress and provide a protective effect against apoptosis (33). Abnormally low expression of $\alpha$ A-crystallins is correlated with PR loss in a rat model of retinal dystrophy (34), and a recent proteomic analysis of the retina in the $r d / r d$ mouse demonstrated induction of crystallin upregulation in response to retinal degeneration (35). Based on our microarray data of $\mathrm{Lin}^{-}$HSC rescued $r d / r d$ mouse retinas, upregulation of crystallins may also play a key role in EPC-mediated retinal neuroprotection.

Genes such as $c-m y c, M a d I, Y y-1$, and the crystallins are likely to be downstream mediators of neuronal rescue. Presumably, neurotrophic agents serve to regulate antiapoptotic gene expression, although our microarray analysis of retinas rescued with mouse stem cells did not demonstrate induction of increased 
levels of known neurotrophic factors. Analysis of hBM-derived stem cell-mediated rescue with human-specific chips did, on the other hand, demonstrate low but significant increases in the expression of multiple growth factor genes. While these data are preliminary, they include several members of the fibroblast growth factor family and otoferlin. Mutations in the otoferlin gene are associated with genetic disorders leading to deafness due to auditory neuropathy $(36,37)$. It is possible that otoferlin production by injected Lin $^{-}$HSCs is critical to the prevention of retinal neuropathy as well.

Historically, it has long been assumed that vascular changes observed in patients and animals with retinal degeneration were secondary to decreased metabolic demand as the PRs die. Our data suggest that, at least for mice with inherited retinal degeneration due to mutations in the cGMP phosphodiesterase gene, preserving normal vasculature correlates with preservation of cones in the ONL as well. Recent reports in the literature would support the concept that tissue-specific vasculature has trophic effects that go beyond that expected from simply providing vascular "nourishment." For example, liver endothelial cells can be induced to produce, after VEGFR1 activation, growth factors critical to hepatocyte regeneration and maintenance in the face of hepatic injury (38). Similar interactions between vascular endothelial cells and adjacent hepatic parenchymal cells are necessary for liver organogenesis, as well as for the formation of functional blood vessels (39). Endogenous retinal vasculature in individuals with retinal degeneration may not facilitate so dramatic a rescue, but perhaps the presence of endothelial progenitors derived from bone marrow hematopoietic stem cell populations makes the vasculature more resistant to degeneration and at the same time facilitates retinal neuronal survival. In humans with this disease, slowing the rate of degeneration, even to a limited extent, may provide years of additional sight. While the animals treated in this study had significant, but not dramatic, preservation of ERG signals, even such minimal signs of electrical function in the retina may be sufficient to support vision.

Clinically, it is widely appreciated that there may be substantial loss of PRs and other neurons while still preserving functional vision. At some point, the critical threshold is crossed and vision is lost. Since nearly all of the human inherited retinal degenerations are of early, but slow, onset, it may be possible to identify an individual with retinal degeneration, treat them intravitreally with an autologous bone marrow stem cell graft, and delay retinal degeneration with concomitant loss of vision. This would be particularly effective if, similar to our observations in the mouse model presented in this study, rescue of the cones would occur. To enhance targeting and incorporation of these stem cells, the presence of activated astrocytes would be desirable (20); this could be accomplished by early treatment when there is an associated gliosis or by using laser to stimulate local proliferation of activated astrocytes. Since the actual mechanism of stem cellmediated rescue remains unknown, ex vivo transfection of the cells with one or several neurotrophic substances prior to intraocular injection may enhance the rescue effect. If the observed neuronal rescue effect is achieved by $\mathrm{Lin}^{-} \mathrm{HSC}$-derived vascular endothelial cell induction of antiapoptotic factor synthesis in a paracrine fashion, it may be possible to apply this approach to the treatment of other visual neuronal degenerative disorders such as glaucoma, in which there is retinal ganglion cell degeneration. Such investigations are currently in progress.

\section{Methods}

Animals. We used C3H/HeJ, C3SnSmn.CB17-Prkdc ${ }^{S C I D}$, and rd10 mouse strains as retinal degeneration models. $\mathrm{C} 3 \mathrm{H} / \mathrm{HeJ}$ and C3SnSmn.CB17-Prkdc SCID (The Jackson Laboratory) are homozygous for the $r d 1$ mutation, and this mutation causes early onset severe retinal degeneration. The mutation is located in exon 7 of the Pde6b gene encoding the rod PR cGMP phosphodiesterase $\beta$ subunit. Mutations in this gene have been found in human patients with autosomal recessive RP (26). C3SnSmn.CB17-Prkdc SCID is also homozygous for the SCID spontaneous mutation (Prkdcsid) and was used for human cell transfer experiments. Retinal degeneration in $r d 10$ mice is caused by a mutation in exon 13 of Pde6b gene. This is also a clinically relevant RP model with later onset and milder retinal degeneration than $r d 1 / r d 1$ (26).

All experiments were performed in accordance with the NIH Guide for the Care and Use of Laboratory Animals, and all experimental procedures were approved by The Scripps Research Institute Animal Care and Use Committee.

Cell preparation. Mouse bone marrow cell extraction was performed as previously reported (20). Briefly, bone marrow cells were harvested from the femurs, tibiae, and humerus bones of each mouse, and mononuclear cells were separated by densitygradient separation using Fico/Lite LM (Atlanta Biologicals Inc.). The mononuclear cells were labeled with biotin-conjugated lineage Ab's (CD45, CD3, Ly-6G, CD11b, TER-119; BD Biosciences - Pharmingen) or CD31 Ab followed by incubation with streptavidin or anti-biotin magnetic beads. Cells were then sorted using the MACS cell-sorting system (Miltenyi Biotec) to obtain Lin- or CD31- cell populations.

Human mononuclear cells (hBM) were obtained from the bone marrow of healthy volunteers. To isolate the $\mathrm{Lin}^{-}$cell population from hBM mononuclear cells the following Ab's were used with the MACS separation system: CD2, CD3, CD4, CD11a, CD11b, CD14, CD16, CD19, CD33, CD38, CD45RA, CD64, CD68, CD86, CD235a (BD Biosciences - Pharmingen). To visualize the injected human cells, cells were labeled with dye (Cell-Tracker Green CMFDA; Invitrogen Corp.) before injection. All studies using hBM conformed to the Scripps Institutional Review Board regulations for research with human subjects.

Intravitreal injection. An eyelid fissure was created with a fine blade to expose the globe of a P6 mouse. Cells (approximately $5 \times 10^{5} /$ $0.5 \mu \mathrm{l}$ ) were injected intravitreally using a 33 -gauge needle (Hamilton Co.). To increase the accuracy of data, Lin $^{-}$cells were injected into one eye and control cells into the contralateral eye of the same animal and resultant effects were compared.

Staining and quantification of retinal vasculature. Retinas were harvested at various time points and fixed with $4 \%$ paraformaldehyde (PFA) and methanol followed by blocking in 50\% FBS/20\% normal goat serum for 1 hour at room temperature. To stain retinal vasculature, retinas were incubated with anti-CD31 (BD Biosciences - Pharmingen) and anti-CIV (Chemicon International) Ab's followed by Alexa 488- or 594-conjugated secondary Ab's (Invitrogen Corp.). The retinas were laid flat with radial relaxing incisions to obtain a whole-mount preparation. Images of vasculature in intermediate or deep retinal vascular plexuses (21) were obtained using a Radiance MP2 100 confocal microscope and LaserSharp software (Bio-Rad Laboratories Inc.). For quantification of vasculature, four independent fields $(900 \mu \mathrm{m} \times 900 \mu \mathrm{m})$ were chosen randomly from the midportion of the intermediate or deep vascular layer, and the total length of vasculature was mea- 
sured using LaserPix analyzing software (Bio-Rad Laboratories Inc.). The total lengths of these four fields in the same plexus were used for further analysis.

Counting the number of cell nuclei in the ONL. The flat-mounted retinas were re-embedded for cryostat sections. Retinas were placed in $4 \%$ PFA overnight followed by incubation with $20 \%$ sucrose. The retinas were embedded in OCT compound (Tissue-Tek; Sakura FineTech Inc.). Cryostat sections $(10 \mu \mathrm{m})$ were rehydrated in PBS containing the nuclear dye DAPI (Sigma-Aldrich). DAPI-labeled nuclear images of three different areas ( $280 \mu \mathrm{m}$ width, unbiased sampling) in a single section that contained the optic nerve head and the entire peripheral retina were taken by confocal microscope. The numbers of nuclei located in the ONL of three independent fields within one section were counted and summed up for analysis.

Simple linear-regression analysis was performed to examine the relationship between the length of vasculature in the deep plexus and the number of cell nuclei in the ONL.

Characterization of rescued $P R$ cell types. Ab's specific for rod rhodopsin (rho4D2) were generously provided by Robert Molday of the University of British Columbia and used as described previously (40). Rabbit Ab's specific for cone red/green opsin were purchased from Chemicon International (AB5405) and used according to the manufacturer's instructions.

Electroretinography. Following overnight dark adaptation, mice were anesthetized by intraperitoneal injection of $15 \mu \mathrm{g} / \mathrm{g}$ ketamine and $7 \mu \mathrm{g} / \mathrm{g}$ xylazine. ERGs were recorded from the corneal surface of each eye after pupil dilation (1\% atropine sulfate) using a gold loop corneal electrode together with a mouth reference and tail ground electrode. Stimuli were produced with a Grass Photic Stimulator (PS33 Plus; Grass Telefactor) affixed to the outside of a highly reflective Ganzfeld dome. Responses were recorded to short-wavelength (Wratten $47 \mathrm{~A} ; 1_{\max }=470 \mathrm{~nm}$ ) flashes of light over a range of intensities up to the maximum allowable by the photic stimulator $\left(0.668 \mathrm{~cd}-\mathrm{s} / \mathrm{m}^{2}\right)$. Response signals were amplified (CP511 AC amplifier; Grass Telefactor), digitized (PCI-1200; National Instruments Corp.), and computer analyzed. Each mouse served as its own internal control with ERGs recorded from both the treated and untreated or control-treated eyes. Up to 100 sweeps were averaged for the weakest signals.

Microarray analysis of stem cell-targeted retinal gene expression. $\mathrm{P} 6 \mathrm{rd} /$ $r d$ mice were injected with either $\mathrm{Lin}^{-}$or CD31- HSCs. The retinas of these mice were dissected 40 days after injection in RNase-free medium (rescue of the retinal vasculature and the PR layer is obvious at this time point after injection). One quadrant from each retina was analyzed by whole mount to ensure that normal HSC targeting as well as vasculature and neural protection had been achieved. RNA from retinas with successful injections was purified using a TRIzol (Life Technologies Inc.) phenol/chloroform RNA isolation protocol. RNA was hybridized to Affymetrix Mu74Av2 chips, and gene expression was analyzed using GeneSpring software (Silicon Genetics).

Purified human or mouse HSCs were injected intravitreally into P6 mice. At P45 the retinas were dissected and pooled into fractions of (a) human HSC-injected rescued mouse retinas, (b) human HSC-injected nonrescued mouse retinas, and (c) mouse HSC-injected rescued mouse retinas for purification of RNA and hybridization to human-specific U133A Affymetrix chips. GeneSpring software was used to identify genes that were expressed above background and with higher expression in the human HSC-rescued retinas. The probe-pair expression profiles for each of these genes were then individually analyzed and compared with a model of normal human U133A microarray experiments using dChip (41) to determine human species-specific hybridization and to eliminate false positives due to cross-species hybridization.

\section{Acknowledgments}

We thank Edith Aguilar and other members of the Friedlander laboratory for assistance and helpful discussions during the course of this study, Matthew Ritter for critical comments on the manuscript, and Maria Concepcion Lillo Delgado of the Scripps Research Institute/University of California, San Diego Eye Core for her expertise in the EM analysis of tissue specimens. We are grateful to Robert Molday of the University of British Columbia for generously providing us with the rod opsin-specific Ab. This work was supported by grants from the National Eye Institute (EY11254 to M. Friedlander), the Robert Mealey Program for the Study of Macular Degenerations, and the Kovner Family Fund.

Received for publication March 23, 2004, and accepted in revised form July 13, 2004.

Address correspondence to: Martin Friedlander, Department of Cell Biology, The Scripps Research Institute, 10550 North Torrey Pines Road, La Jolla, California 92037, USA. Phone: (858) 784-9138; Fax: (858) 784-9135; E-mail: friedlan@scripps.edu.

John Heckenlively's present address is: Kellogg Eye Center, University of Michigan, Ann Arbor, Michigan, USA.

Atsushi Otani's present address is: Department of Ophthalmology, Kyoto University, Kyoto, Japan.
1. Heckenlively, J.R., editor. 1988. Retinitis pigmentosa. J.B. Lippincott Co. Philadelphia, Pennsylvania, USA.

2. Humphries, P., Kenna, P., and Farrar, G.J. 1992. On the molecular genetics of retinitis pigmentosa. Science. 256:804-808.

3. Farrar, G.J., Kenna, P.F., and Humphries, P. 2002. On the genetics of retinitis pigmentosa and on mutation-independent approaches to therapeutic intervention. EMBO J. 21:857-864.

4. Dryja, T.P., et al. 1990. A point mutation of the rhodopsin gene in one form of retinitis pigmentosa. Nature. 343:364-366.

5. Bowes, C., et al. 1990. Retinal degeneration in the rd mouse is caused by a defect in the beta subunit of rod cGMP-phosphodiesterase. Nature. 347:677-680.

6. Kajiwara, K., et al. 1991. Mutations in the human retinal degeneration slow gene in autosomal domi- nant retinitis pigmentosa. Nature. 354:480-483.

7. Gu, S.M., et al. 1997. Mutations in RPE65 cause autosomal recessive childhood-onset severe retinal dystrophy. Nat. Genet. 17:194-197.

8. Ali, R.R., et al. 2000. Restoration of photoreceptor ultrastructure and function in retinal degeneration slow mice by gene therapy. Nat. Genet. 25:306-310.

9. Takahashi, M., Miyoshi, H., Verma, I.M., and Gage, F.H. 1999. Rescue from photoreceptor degeneration in the rd mouse by human immunodeficiency virus vector-mediated gene transfer. J. Virol. 73:7812-7816.

10. Acland, G.M., et al. 2001. Gene therapy restores vision in a canine model of childhood blindness. Nat. Genet. 28:92-95.

11. Frasson, M., et al. 1999. Retinitis pigmentosa: rod photoreceptor rescue by a calcium-channel blocker in the rd mouse. Nat. Med. 5:1183-1187.

12. Frasson, M., et al. 1999. Glial cell line-derived neurotrophic factor induces histologic and functional protection of rod photoreceptors in the $\mathrm{rd} / \mathrm{rd}$ mouse. Invest. Ophthalmol. Vis. Sci. 40:2724-2734.

13. Berson, E.L., et al. 1993. A randomized trial of vitamin A and vitamin E supplementation for retinitis pigmentosa. Arch. Ophthalmol. 111:761-772.

14. Mohand-Said, S., et al. 1998. Normal retina releases a diffusible factor stimulating cone survival in the retinal degeneration mouse. Proc. Natl. Acad. Sci.U.S. A. 95:8357-8362.

15. Asahara, T., et al. 1997. Isolation of putative progenitor endothelial cells for angiogenesis. Science. 275:964-967.

16. Kalka, C., et al. 2000. Vascular endothelial growth factor (165) gene transfer augments circulating 
endothelial progenitor cells in human subjects. Circ. Res. 86:1198-1202.

17. Gill, M., et al. 2001. Vascular trauma induces rapid but transient mobilization of VEGFR2(+)AC133(+) endothelial precursor cells. Circ. Res. 88:167-174.

18. Kocher, A.A., et al. 2001. Neovascularization of ischemic myocardium by human bone-marrow-derived angioblasts prevents cardiomyocyte apoptosis, reduces remodeling and improves cardiac function. Nat. Med. 7:430-436.

19. Grant, M.B., et al. 2002. Adult hematopoietic stem cells provide functional hemangioblast activity during retinal neovascularization. Nat. Med. 8:607-612.

20. Otani, A., et al. 2002. Bone marrow-derived stem cells target retinal astrocytes and can promote or inhibit retinal angiogenesis. Nat. Med. 8:1004-1010.

21. Dorrell, M.I., Aguilar, E., and Friedlander, M. 2002. Retinal vascular development is mediated by endothelial filopodia, a preexisting astrocytic template and specific R-cadherin adhesion. Invest Ophthalmol. Vis. Sci. 43:3500-3510.

22. Chang, G.Q., Hao, Y., and Wong, F. 1993 Apoptosis: final common pathway of photoreceptor death in rd, rds, and rhodopsin mutant mice. Neuron. 11:595-605.

23. Carter-Dawson, L.D., LaVail, M.M., and Sidman, R.L. 1978. Differential effect of the rd mutation on rods and cones in the mouse retina. Invest. Ophthal mol. Vis. Sci. 17:489-498.

24. Blanks, J.C., and Johnson, L.V. 1986. Vascular atrophy in the retinal degenerative rd mouse. J. Comp. Neurol. 254:543-553.
25. Soucy, E., Wang, Y., Nirenberg, S., Nathans, J., and Meister, M. 1998. A novel signaling pathway from rod photoreceptors to ganglion cells in mammalian retina. Neuron. 21:481-493.

26. Chang, B., et al. 2002. Retinal degeneration mutants in the mouse. Vision Res. 42:517-525.

27. Tu, Y., Stolovitzky, G., and Klein, U. 2002. Quantitative noise analysis for gene expression microarray experiments. Proc. Natl. Acad. Sci. U. S. A. 99:14031-14036.

28. Austen, M., et al. 1997. Regulation of cell growth by the Myc-Max-Mad network: role of Mad proteins and YY1. Curr. Top. Microbiol. Immunol. 224:123-130.

29. Cortes, F., et al. 1999. HCA, an immunoglobulin-like adhesion molecule present on the earliest human hematopoietic precursor cells, is also expressed by stromal cells in blood-forming tissues. Blood. 93:826-837.

30. Van Pesch, V., and Michiels, T. 2003. Characterization of interferon-alpha 13, a novel constitutive murine interferon-alpha subtype. J. Biol. Chem. 278:46321-46328.

31. Dang, C.V. 1999. c-Myc target genes involved in cell growth, apoptosis, and metabolism. Mol. Cell. Biol. 19:1-11

32. Gehring, S., et al. 2000. Inhibition of proliferation and apoptosis by the transcriptional repressor Mad1. Repression of Fas-induced caspase-8 activation. J. Biol. Chem. 275:10413-10420

33. de Jong, W.W., Hendriks, W., Mulders, J.W., and Bloemendal, H. 1989. Evolution of eye lens crys- tallins: the stress connection. Trends Biochem. Sci. 14:365-368.

34. Maeda, A., Ohguro, H., Maeda, T., Nakagawa, T., and Kuroki, Y. 1999. Low expression of alphaAcrystallins and rhodopsin kinase of photoreceptors in retinal dystrophy rat. Invest. Ophthalmol. Vis. Sci. 40:2788-2794.

35. Cavusoglu, N., et al. 2003. Differential proteomic analysis of the mouse retina: the induction of crystallin proteins by retinal degeneration in the rd1 mouse. Mol. Cell Proteomics. 2:494-505.

36. Varga, R., et al. 2003. Non-syndromic recessive auditory neuropathy is the result of mutations in the otoferlin (OTOF) gene. J. Med. Genet. 40:45-50.

37. Riazuddin, S., et al. 2000. Dominant modifier DFNM1 suppresses recessive deafness DFNB26. Nat. Genet. 26:431-434.

38. LeCouter, J., et al. 2003. Angiogenesis-independent endothelial protection of liver: role of VEGFR-1. Science. 299:890-893.

39. Matsumoto, K., Yoshitomi, H., Rossant, J., and Zaret, K.S. 2001. Liver organogenesis promoted by endothelial cells prior to vascular function. Science. 294:559-563.

40. Hicks, D., and Molday, R.S. 1986. Differential immunogold-dextran labeling of bovine and frog rod and cone cells using monoclonal Ab's against bovine rhodopsin. Exp. Eye Res. 42:55-71.

41. Li, C., and Wong, W.H. 2001. Model-based analysis of oligonucleotide arrays: expression index computation and outlier detection. Proc. Natl. Acad. Sci. U. S. A. 98:31-36. 